\title{
D'une initiative locale à un essaimage national, l'histoire du développement du maïs en France
}

From a local initiative to a national expansion: the history of the developement of maize in France

De lo local a lo nacional: historia de las variedades no híbridas de maíz en Francia

\section{AgroBio Périgord}

\section{OpenEdition}

Journals

Édition électronique

URL : https://journals.openedition.org/ethnoecologie/7745

DOI : $10.4000 /$ ethnoecologie. 7745

ISSN : 2267-2419

Éditeur

Laboratoire Éco-anthropologie

Référence électronique

AgroBio Périgord, « D'une initiative locale à un essaimage national, l'histoire du développement du maïs en France », Revue d'ethnoécologie [En ligne], Supplément 2 | 2021, mis en ligne le 25 novembre 2021, consulté le 20 décembre 2021. URL : http://journals.openedition.org/ethnoecologie/7745; DOI : https://doi.org/10.4000/ethnoecologie.7745

Ce document a été généré automatiquement le 20 décembre 2021.

\section{(c) $($ ) $(9)$}

Revue d'ethnoécologie est mis à disposition selon les termes de la licence Creative Commons Attribution - Pas d'Utilisation Commerciale - Pas de Modification 4.0 International. 


\section{D'une initiative locale à un essaimage national, l'histoire du développement du maïs en France}

From a local initiative to a national expansion: the history of the developement of maize in France

De lo local a lo nacional: historia de las variedades no híbridas de maíz en Francia

AgroBio Périgord

\section{Introduction}

1 Au cours de la deuxième moitié $\mathrm{du} \mathrm{xx}^{\mathrm{e}}$ siècle, le maïs en France est devenu une plante emblématique de la modernisation de l'agriculture et du développement des technologies hybrides. Le gouvernement décourage les agriculteurs à sélectionner, multiplier et conserver eux-mêmes les semences et ces tâches sont de plus en plus confiées à des professionnels publics puis aux entreprises privées. À partir des années 1960 l'achat de semences commerciales se généralise en France. Les variétés locales de maïs disparaissent quasiment toutes au cours des trente glorieuses pour être remplacées par le maïs hybride.

2 À la fin des années 1990, le thème des semences de maïs anime le débat français et européen concernant les nouvelles réglementations en matière d'OGM. «En février 1998, la France fut le premier pays européen à autoriser la culture de maïs transgénique. Cet événement réveilla les consciences. Dans ce contexte, l'arrivée des OGM sur le marché a mis en lumière le risque de voir remplacées les semences classiques par des semences OGM. » (Collectif 2015). Cette ouverture législative au maïs OGM et le problème des possibles contaminations des semences de maïs hybride ont été un électrochoc pour plusieurs agriculteurs et associations. Le risque de contamination des semences commerciales conduit les agriculteurs, surtout en agriculture biologique, 
à chercher des systèmes pour maîtriser l'origine de leurs semences afin de sécuriser leur production. Cette situation porte à l'émergence d'initiatives visant le développement des semences paysannes ${ }^{1}$ de maïs en France. Pour caractériser ce travail, basé sur des semences de maïs non hybrides, les agriculteurs et les associations ont décidé d'utiliser le nom «maïs population " en faisant référence à la notion de variété de population. Celle-ci est une variété hétérogène car constituée d'un ensemble d'individus génétiquement différents. Les populations sont multipliées en pollinisation libre et sélectionnées par les agriculteurs eux-mêmes. À la différence du maïs hybride qui, lorsqu'il est ressemé, perd son potentiel productif dans les générations successives, les variétés Population peuvent être ressemées d'une année sur l'autre. Ces semences «reproductibles» donnent une plus grande autonomie aux agriculteurs et leur permettent d'améliorer certaines caractéristiques du maïs ainsi que de l'adapter à leur condition de culture.

3 Bertrand Lassaigne est producteur en agriculture biologique en Dordogne. En 2000, il était administrateur de la structure de développement de l'agriculture biologique départementale et autoproduisait la quasi-totalité des semences de ses cultures, sauf pour le maïs.

«En 2000, un agriculteur bio ayant acheté des semences non traitées de maïs s'est rendu compte que celles-ci étaient contaminées par des OGM. J'ai alors été pris de doute quant à ma propre production. La nécessité de trouver une alternative aux semences proposées par les semenciers auxquels je ne faisais plus confiance est devenue une priorité. J'ai donc décidé de chercher des variétés de maïs non hybrides datant d'avant la révolution verte. Je suis alors parti au Guatemala, où les paysans locaux ressèment leurs propres variétés. » (Bio d'Aquitaine 2001).

Bertrand ramena de son voyage 11 variétés guatémaltèques de maïs, qu'il sema dans ses champs au printemps suivant, sous forme d'une plateforme. C'est donc la conjoncture $\mathrm{du}$ contexte français particulièrement bouillonnant et le voyage au Guatemala de Bertrand Lassaigne qui marquent le début symbolique du développement du Maïs Population en France.

\section{Une volonté de partager et de faire bénéficier largement de l'expérience, à l'image de la plante maïs, allogame}

\footnotetext{
«Au commencement, la majorité des gens me prenait pour un fou [...] seule une poignée de producteurs d'AgroBio Périgord trouvèrent cette démarche intéressante et l'association a alors décidé d'accompagner le développement de ce projet». (Bertrand Lassaigne 2011)
}

6 Un premier budget fut trouvé en 2001 et un salarié de l'association AgroBio Périgord travailla à mi-temps sur cette thématique du développement des Maïs Population. Le programme régional «l'Aquitaine cultive la biodiversité" fut créé, et la parcelle "plateforme", avec les 11 variétés guatémaltèques ouvrit ses portes à l'automne. Un travail de communication a ensuite été mené autour de ces travaux afin de leur donner de la résonnance.

7 Une journée de formation et de visite de la plateforme de culture de ces variétés de maïs venues d'ailleurs a été organisée, permettant ainsi la rencontre d'AgroBio 
Périgord avec des personnes isolées, qui cultivaient individuellement des variétés de maïs venant de leurs aïeux ou bien d'autres pays en Europe. Cette journée a également attiré des personnes intéressées pour voir et essayer ces variétés « reproductibles » qui présentaient des caractéristiques telles que la couleur, la taille de l'épi et de la plante, très différentes de ce que l'on pouvait voir habituellement en France.

C'est ainsi, et par le bouche-à-oreille, que la dynamique collective naquit grâce à la curiosité des agriculteurs et à leur intérêt pour ces variétés si différentes de celles qu'ils avaient l'habitude de voir dans le commerce. La participation grandissante d'agriculteurs se révéla bientôt être une condition fondamentale pour le développement du projet. En effet, afin de permettre la multiplication des populations de maïs et la production de semences en quantité suffisante pour pouvoir cultiver et conserver ces variétés de manière pérenne, il a fallu mettre en place une gestion collective des semences produites. Ceci s'explique par le mode de reproduction du maïs, qui est une plante allogame: le pollen des fleurs d'une plante est dispersé souvent avant que les fleurs femelles de cette plante ne soient prêtes à être fécondées. Elle se féconde donc peu elle-même. Le fait de cultiver différentes variétés en un seul lieu, comme cela peut se faire pour les céréales à paille, est donc rédhibitoire car il rend impossible le contrôle de la pollinisation et permet alors aux différentes variétés de se croiser entre elles, ce qui nuit à la conservation de leur patrimoine génétique. Le mode de reproduction du maïs a obligé à mettre en place une gestion collective des semences qui permettait de conserver les différentes variétés sur des fermes différentes en minimisant ainsi les risques de croisements. La nécessité de répartir les variétés et les responsabilités de conservation et de multiplication a conduit à la mise en place de dispositifs de gestion collective de la diversité du maïs. Ce mode de fonctionnement fut rapidement élargi au-delà du département de la Dordogne. Toute demande de semence était la bienvenue et un système très flexible de gestion des semences en réseau a été depuis le début expérimenté.

En 2002, la plateforme fut reconduite avec les variétés désormais disponibles telles que les variétés guatémaltèques ayant pu arriver à maturité et produire suffisamment de graines, les variétés collectées par le bouche-à-oreille, ainsi que certaines accessions sorties du Centre de Ressources Biologiques (CBR) du Maïs de Montpellier ${ }^{2}$. Depuis son début, le rôle de la vitrine-plateforme en Dordogne n'a pas cessé d'évoluer. Conçue initialement comme support de sensibilisation et de communication pour les agriculteurs sur le thème du Maïs Population, sa fonction a été au fur et à mesure adaptée selon des nouveaux besoins. Il était notamment urgent de produire des connaissances sur les variétés en mettant en place un travail de caractérisation. En effet, il était primordial de pouvoir définir leurs caractéristiques principales, telles que la précocité, pour pouvoir les diffuser. L'enjeu était majeur : il fallait répondre à des demandes de plus en plus nombreuses et précises.

10 Parallèlement à la systématisation des informations sur la précocité des différentes variétés de maïs, d'autres informations sont devenues nécessaires telles que le rendement du maïs grain, le développement végétatif ou encore les caractéristiques nutritionnelles du grain (farineux ou vitreux, taux de protéines, matières grasses, sucres et amidon).

11 La production et la transmission des connaissances sur les variétés est rapidement devenue une des principales missions de l'équipe d'Agrobio Périgord impliquée sur le Projet Maïs. AgroBio Périgord réalise chaque année un travail de compilation de 
données sur les variétés de Maïs Population cultivées et gère plusieurs essais expérimentaux (RSP et al. 2009, RSP 2014). Tous les ans, un rapport est distribué lors de la visite annuelle de la plateforme, présentant les résultats des suivis de l'année précédente (AgroBio Périgord 2003-2020). Des ouvrages plus génériques ont également été édités en partenariat avec d'autres structures (RSP ${ }^{3}$, INRA $\left.{ }^{4}, \mathrm{ITAB}^{5} . ..\right)$.

\section{Une officialisation rapide de la démarche dans un contexte réglementaire pourtant incertain}

12 C'est auprès du Conseil Régional d'Aquitaine que le premier soutien financier fut trouvé, puis rapidement, il fut accompagné de fonds de développement européen. Dès lors, une condition sine qua non fut portée par les financeurs : il fallait pouvoir prouver l'expertise du travail de sélection mené par AgroBio Périgord. En effet, un groupe d'agriculteurs, seul, n'avait pas la légitimité requise face aux regards extérieurs ainsi qu'au monde de la sélection et de la création variétale. Les notions de recherche participative et de création de projet «bottom-up » n'étaient pas encore dans l'air du temps. Un partenariat avec un sélectionneur indépendant, Guy Thiébaut, fut alors mis en place. Il permit d'effectuer un long travail de création et d'amélioration variétale sur la base des méthodes «hybrides », mais en ayant comme principe ici des croisements frères-sœurs entre populations et non entre lignées.

13 Ce travail ne fut pas uniquement mis en place pour répondre aux financeurs, il répondait à l'époque à une demande provenant des agriculteurs eux-mêmes. De nombreuses variétés locales récupérées avaient souffert d'un processus de dégénérescence. D'autres, surtout celles provenant de pays tropicaux, s'étaient révélées encore inadaptées au climat. Il était donc nécessaire et urgent de consacrer une partie du travail à la régénération et à l'adaptation de ces variétés. Bertrand Lassaigne participera grandement à cette phase en réalisant lui-même des mélanges de différentes populations en croisements libres qui ont donné des nouvelles créations variétales telles que le Lavergne et le Bénastone. L'émergence de ces initiatives se produit dans un contexte réglementaire qui n'est pourtant pas favorable à la libre circulation des semences et des plants destinés aux exploitations commerciales. À partir de la seconde moitié $\mathrm{du} \mathrm{xx}^{\mathrm{e}}$ siècle, parallèlement à la professionnalisation du métier du sélectionneur et au développement de la filière des semences commerciales, un cadre normatif, visant à protéger les droits de propriété intellectuelle, voit le jour. En France la mise en place des Certificats d'Obtention Végétale (COV) en 1961 vise à aligner le marché des semences aux normes de Distinction, d'Homogénéité et de Stabilité (DHS) exigées par le catalogue. Bien qu'il s'agisse d'un dispositif plus souple du brevet, il permet un premier verrouillage du commerce de semences en France. À partir des années 1970, en accord avec les nouvelles normes de l'Union pour la Protection des Obtentions Végétales, les semences de ferme sont interdites dans les productions à destination commerciale, à l'exception de quelques espèces, pour lesquelles une dérogation est mise en place en 1994, en contrepartie du paiement de royalties.

14 À la fin des années 1990, la communauté européenne a commencé à débattre d'une série de directives pour développer des politiques semencières plus adaptées à l'agriculture biologique et pour mettre en place des mécanismes pour la conservation d'espèces agricoles menacées d'érosion génétique. En avril 2018, le parlement européen a adopté un nouveau règlement sur l'agriculture biologique qui entrera en vigueur le 
$1^{\mathrm{er}}$ janvier 2021. Ce règlement permet la commercialisation des variétés qui ne répondent pas aux critères DHS (distinction, homogénéité et stabilité) 6 . La possibilité de commercialiser $\mathrm{du}$ «matériel hétérogène biologique » et, pas simplement de l'utiliser pour l'expérimentation variétale, a ouvert un espace légitime pour la diffusion de semences paysannes mais il a provoqué en même temps des craintes. Il pourrait y avoir la possibilité que ce nouveau règlement puisse être utilisé par les firmes pour commercialiser les nouvelles variétés obtenues avec les nouvelles techniques de sélection végétale tel que le CRISPR-Cas9.

\section{À l'origine d'une structure nationale sur les semences paysannes}

L'initiative lancée sur les Maïs Population en Dordogne est loin d'être un cas marginal et isolé. D'autres actions similaires voient le jour, discrètement, un peu partout en France. Face au contexte réglementaire lourd et hostile, chacun se sent fragile à porter des projets sur les semences paysannes, pourtant foncièrement légitimes : recourir aux droits fondamentaux des paysans à semer et ressemer leurs propres semences. Pour soutenir la démarche commencée en Dordogne, les paysans ou groupements, comme Bertrand Lassaigne et AgroBio Périgord se rapprochent alors de différentes structures telles que la Confédération Paysanne, la Coordination Nationale de Défense des Semences de Ferme (CNDSF), la Fédération Nationale de l'Agriculture Biologique (FNAB), le Mouvement de Culture Biodynamique (MCBD), Nature et Progrès (N\&P)).

16 En février 2003, dans le cadre des premières rencontres "Semences Paysannes, cultivons la biodiversité dans les fermes » à Auzeville, la Confédération Paysanne et la FNAB présentent un état des lieux des projets individuels et collectifs de conservation, multiplication et sélection de semences à la ferme. Ces rencontres rassemblent près de 350 personnes. Elles aboutissent à la rédaction d'une déclaration qui engage l'ensemble des participants et les structures organisatrices à défendre les droits fondamentaux des paysans vis-à-vis de la semence et à construire une alternative collective aux variétés industrielles. Ce jour marque une étape fondamentale dans la lutte pour la sauvegarde de l'accès des semences paysannes et celles non enregistrées au catalogue officiel des semences.

« Pour moi Auzeville restera le souvenir fort de la rencontre entre des paysans qui travaillaient chacun dans leur coin à la sélection de semences paysannes et réalisent avec émotion que d'autres, comme eux, y œuvrent d'arrache-pied. Ils ne sont plus seuls! Ce fut aussi le souvenir de la rencontre de chercheurs avec un monde paysan a priori méfiant vis-à-vis des scientifiques... A se découvrir des objectifs communs, on s'organise pour travailler ensemble et chacun apprend des autres! Dernier moment fort pour moi : la prise de conscience collective que le monde paysan s'est laissé déposséder à travers le temps d'un certain nombre de prérogatives, notamment dans le domaine des semences et qu'il est nécessaire d'y remédier. Ensemble. on cherche des solutions techniques certes, mais l'aventure reste bien humaine...» (Marie-Paule Hernandez, agricultrice bio en grandes cultures avec transformation dans les Landes, impliquée dans le programme L'Aquitaine cultive la biodiversité depuis 2003).

À l'issue de ces rencontres, le Réseau Semences Paysannes se constitue en 2004 et aujourd'hui, 14 ans plus tard, il regroupe près de 90 organisations dans toute la France 
et sur plusieurs espèces. Il est en lien étroit avec d'autres réseaux européens tels que la Rete Semi Rurali en Italie...) et fait partie du réseau européen Let's Liberate Diversity.

La création de ce réseau à échelle nationale permet de rassembler des collectifs autour d'idées et de problématiques qui étaient auparavant traitées individuellement et sans mutualisation. Cela permet la mise en commun de résultats et l'organisation à plus grande ampleur d'actions cherchant à répondre aux problématiques rencontrées par les paysans. C'est par exemple ainsi que le voyage d'étude en 2004 au Brésil s'est organisé. Il avait pour objectif de répondre aux questions portant sur les méthodes de sélection à la portée des paysans, ainsi que sur les modes d'organisation pour la gestion des variétés paysannes de maïs.

\title{
Un fonctionnement interne déterminé par le mode de reproduction de la plante maïs
}

\begin{abstract}
«Il est presque impossible pour un agriculteur de conserver, sélectionner et produire seul toutes ses semences, ni de ressemer ses champs si l'année précédente a été trop mauvaise. Aucune sélection n'est durable sans permettre à la semence de renouveler sa diversité, en changeant de terrain de culture, ou par de petits apports exogènes : il n'y a pas de semences Population sans échanges et sans travail collectif. Les maisons de la semence, qui émergent un peu partout dans le monde, sont l'outil de cette organisation collective entre paysans et jardiniers. Entre échanges de semences et de savoir-faire, elles permettent de répondre aux besoins organisationnels (questions techniques, sociales, etc.) nécessaires au développement des semences paysannes quand le cadre réglementaire tend à les interdire » (RSP 2014).
\end{abstract}

Lors d'un voyage d'échange d'expériences réalisé au Brésil en 2004, les responsables du programme L'Aquitaine cultive la biodiversité rencontrent différentes communautés ayant mis en place des collectifs locaux d'échange et de stockage de semences traditionnelles ou issues de sélection participative. Ce sont ces dispositifs, appelés au Brésil casa de sementes (littéralement "maison des semences ») qui servent de source d'inspiration pour la mise en place d'un autre projet, celui de la Maison de la Semence en Aquitaine. (FNAB 2014)

Dès le début, une des questions principales qui se posent concerne la gestion des semences, c'est-à-dire les demandes et les retours des semences à la Maison de la Semence. AgroBio Périgord s'occupe ainsi de gérer les échanges dans le respect de certaines conditions. Il faut par exemple signer une convention d'expérimentation et faire un retour de semence. De fait, sans retour de semence, il ne peut y avoir de gestion collective avec une plante allogame telle que le maïs, car elle nécessite un isolement de 200 à $400 \mathrm{~m}$ pour éviter les pollinisations croisées. L'animateur-technicien salarié centralise les échanges des semences : après prise en compte des demandes, il gère les lots des semences destinées à l'échange (réception et gestion des retours de semences, envois, traçabilité, analyses) et s'occupe des questions administratives liées à la convention d'expérimentation.

21 Par ailleurs, une restitution de semences est essentielle afin de pouvoir gérer collectivement la semence. Cela permet de constituer un stock disponible pour l'année culturale suivante, et qui sera diffusé à d'autres agriculteurs. Ce retour de semences est une forme d'implication et de responsabilisation de chaque agriculteur, et permet de l'inscrire dans une démarche participative. Chaque agriculteur s'intègre au collectif et 
voit son travail comme une brique apportée à l'édifice en construction qu'est la conservation et la caractérisation des variétés gérées par la Maison de la Semence.

La quantité de semences qui est donnée à chaque agriculteur (lot) permet d'emblaver au départ une $10^{\text {aine }}$ d'ares $\left(=1000 \mathrm{~m}^{2}\right)$. Ces quantités ont par la suite évolué pour arriver à un maximum de $12 \mathrm{~kg}$, soit 0,5 ha semé à une densité de 75000 pieds/ha. AgroBio Périgord ne souhaite pas fournir une plus grande quantité de semences pour trois raisons principales. D'abord le stock de semences d'AgroBio est, pour des raisons techniques et d'espace, limité à des petites quantités pour chaque variété, ensuite la quantité disponible chaque année peut varier en fonctions des retours des agriculteurs de l'année précédente. Enfin, l'association encourage les agriculteurs partenaires à adopter une attitude prudente vis-à-vis de la culture de Maïs Population. En effet, les variétés Populations ont une productivité moins prédictible que les hybrides, et leur adaptation au nouvel environnement dans lequel elles sont implantées est incertaine. Ainsi il est nécessaire qu'un agriculteur teste la variété qui lui a été confiée pendant plusieurs années afin de confirmer qu'elle est bien adaptée à son terroir. Le choix de la variété pour chaque agriculteur fait donc partie intégrante du processus expérimental de culture.

«Les expérimentations effectuées dans les exploitations permettent de tester les semences dans des milieux différents, de sélectionner celles qui ont résisté aux maladies et dont la vigueur est satisfaisante ou qui répondent favorablement aux contraintes spécifiques d'un écosystème. Elles permettent donc de promouvoir des variétés qui correspondent aux besoins des agriculteurs... » (FRB 2011)

AgroBio Périgord bénéficie d'une structure aménagée permettant aux salariés d'assurer la conservation des différentes variétés ainsi que leur diffusion aux agriculteurs. Mais au-delà de la présence de ce lieu physique, l'association souligne que la dénomination Maison des Semences renvoie avant tout au concept de gestion collective des semences. À la base du fonctionnement de la Maison de la Semence, il y a l'organisation en réseau. Il est primordial de "penser" pour le collectif : cela signifie que la semence doit être gardée non seulement pour soi mais aussi pour les besoins du collectif car une perte peut arriver à tout moment. C'est donc dans ces principes que réside la force du collectif.

Pendant les 15 ans suivant la création de la Maison de la Semence, un groupe d'agriculteurs du département de la Dordogne se réunissait annuellement afin de faire le bilan de la campagne de l'année et de discuter de projets pour les années à venir. La priorité était consacrée à l'acquisition de connaissances sur les variétés et leurs comportements via l'expérimentation chez les paysans, en plateforme ou in situ. Malgré le peu de temps accordé à l'animation locale, ce groupe s'est tout de même développé et compte aujourd'hui une quarantaine de fermes sur le département. La volonté actuelle du groupe est de travailler sur l'animation et d'organiser des temps forts tout au long de l'année.

\section{L'inspiration brésilienne à la base des principes de sélection paysanne}

Une grande quantité de variétés de Maïs Population, tant locales que provenant d'Amérique Latine ou encore d'Europe, ont été collectées par les différents membres du programme L'Aquitaine cultive la biodiversité. Cependant ces derniers ont rapidement été 
confrontés à leur manque de connaissances des populations de maïs. En effet, la question d'acquérir des nouvelles pratiques de sélection n'était pas encore résolue. En France, les savoir-faire sur la sélection variétale à la ferme, avaient quasiment tous disparu et les conseils des anciens dont les collectifs pouvaient bénéficier (tels que prendre les plus beaux épis du champ, enlever la pointe et la base de l'épi...) se révélaient insuffisants vu l'ampleur du travail engagé.

Le voyage d'étude organisé par le Réseau Semences Paysannes en 2004 au Brésil apporta des éclairages sur un certain nombre de questions telles que les notions de nombre minimum d'individus, de diversité et de dégénérescence, d'observation des plantes au champ, de densité des plantes sélectionnées, de critères de sélection, de relation de cause à effet entre caractéristiques et problématiques (hauteur d'insertion d'épi et verse des épis...).

Les informations et les connaissances recueillies lors de ce voyage ont ensuite été relayées et diffusées par les collectifs lors de la distribution de semences aux agriculteurs. Les agriculteurs ont alors appliqué les méthodes de sélection amenées du Brésil, et peu à peu, ils se sont appropriés ces pratiques. Cette appropriation progressive des méthodes de sélection et les connaissances sur la sélection participative ont été étayées grâce à la collaboration depuis 2014 avec des chercheurs et techniciens mexicains, spécialistes en la matière (Figure 1).

«Aujourd'hui, je ne suis plus vraiment le protocole [donné par AGP]. Certains agriculteurs invitent des copains, et c'est comme pour les battages autrefois, moi je le fais seul pour mes 5 ha. Je laisse un peu parler le feeling pour faire ma sélection. [...] Il y a quelque chose de visuel, un peu instinctif. Sans ouvrir, je touche l'épi à travers les feuilles, et puis je regarde l'aspect général de la plante. Sur les cinq hectares, j'ai trois variétés de maïs et je sélectionne sur la totalité de mes parcelles. Je passe tous les deux ou quatre rangs en fonction de la configuration et je ramasse sur toute la longueur des rangs. [...] ça me prend en moyenne une demi-journée en marchant bien. Mais j'aime bien, il n'y a pas de bruit dans les maïs, c'est un moment où on peut vraiment observer. Il $\mathrm{y}$ a un rapport particulier avec la culture. [...] Ensuite, je les ramène à l'exploitation, je les mets dans des filets à pomme de terre, une trentaine dans chaque, que je suspends sous un hangar ouvert côté est. Je laisse sécher jusqu'en mars, et j'égraine. Depuis deux ans, je n'enlève plus la tête et le bas de l'épi, je mets tout l'épi » (Armand Duteil 2014).

«Nous, on se met à six ou sept producteurs du coin, et on fait de la sélection massale positive sur 30 ares, qui nous sert à fabriquer de la semence. On sème ces semences l'année suivante sur deux hectares, que l'on moissonne au Corn-Picker, sans sélection. Ça nous permet de semer en troisième année, 70 ha de population. Et bien sûr ces 70 ha, on les ensile pour le troupeau, mais on n'en fait pas de semence. Les deux hectares, nous donnent $50 \mathrm{~kg}$ de semences pour chacune des quatre variétés que l'on cultive. Pour faire la sélection massale, on se retrouve à 30 : on invite des amis, des voisins. C'est un moment convivial. On y passe une demijournée. Comme consigne, on demande aux gens de regarder cinq ou six critères : l'allure générale de la plante, la non-contamination par la fusariose ou d'autres maladies, la hauteur de l'épi [épi trop haut = pied fragilisé] et la précocité. Il ne faut pas trop de critères, car sinon ça devient compliqué à expliquer. L'objectif c'est d'avoir entre 500 et 600 épis sur 30 ares et de ne pas les prendre tous au même endroit pour laisser de la diversité. [...] On fait comme ça, parce qu'on a beaucoup de surface. L'idéal serait de tout faire en sélection massale positive, mais ça nous prendrait trop de temps. Puis, ça fonctionne assez bien. Surtout qu'on ensile pour la consommation animale, c'est satisfaisant ». (Bruno Joly 2014) 
Figure 1 : Journées Franco-mexicaines à Périgueux en 2014. Pendant une visite d'une parcelle, chaque participant (agriculteurs, chercheurs, techniciens) a récolté la plante de maïs qui rassemblait le mieux les caractéristiques qu'il souhaitait et a ensuite expliqué les raisons de son choix aux autres

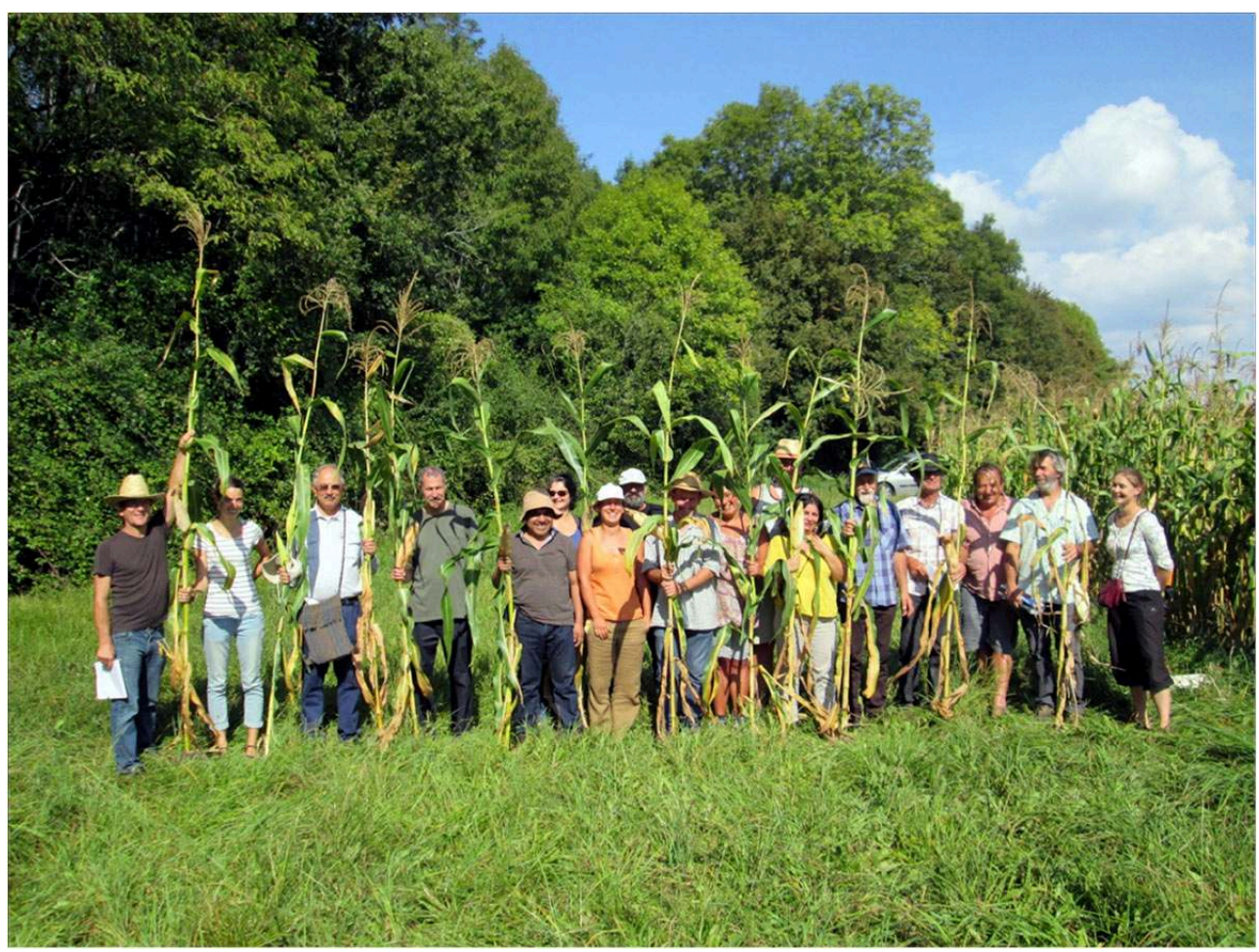

\section{Le début de l'essaimage, en région puis hors des " frontières " administratives avec un élargissement hors de la bio}

Depuis le début de ce programme, les agriculteurs ont exprimé des demandes concernant la mise en place de formations spécifiques sur la culture du Maïs Population. Des modules de formations ont alors été conçus pour transmettre les connaissances acquises sur les variétés, ainsi les savoir-faire en sélection inspirés des échanges avec les Brésiliens et les Mexicains. Cela permet en outre de replacer la démarche dans le contexte réglementaire en vigueur en France. Aujourd'hui, ce sont en tout près de 80 formations qui ont été dispensées par AgroBio Périgord dans de nombreuses structures en France.

En 2005, une situation nouvelle se présente à l'équipe d'Agrobio Périgord: un collectif de paysans conventionnels (non bio) demande une intervention, en exprimant un intérêt pour le Maïs Population. La réalisation de cette intervention qui au début semblait problématique, a permis de rencontrer un groupe de producteurs extrêmement motivés, avec une dynamique collective déjà existante et prête à porter localement le concept de gestion collective de la biodiversité. Cette rencontre a amorcé le développement des Maïs Population hors des frontières de l'agriculture biologique et a mis en lumière les valeurs fédératrices du projet: "transmettre des semences reproductibles de variétés évolutives et les savoir-faire associés ainsi que le concept de gestion collective dynamique ». 


\title{
De multiples axes de recherche et de développement en sélection participative
}

\author{
«Depuis 2007, AgroBio Périgord commence également à s'investir dans des \\ programmes de recherche publique officialisant ainsi un certain nombre de liens \\ déjà existants avec des chercheurs (V. Chables, INRA, D. Desclaux, INRA). Des \\ financements sont alors obtenus pour mettre en place des expérimentations sur des \\ questions spécifiques, sur la plateforme d'expérimentation, ou directement chez les \\ producteurs » (Collectif 2015).
}

Ces projets tournent autour des questions de sélection, de valorisation des Maïs Population en alimentation humaine, de comparaison entre les modes de conservation ex-situ et in-situ, de notions d'évolution et d'adaptation des variétés, de qualités nutritionnelles des variétés (pour l'alimentation du bétail ou humaine...), de création variétale...

Ils s'inscrivent dans des programmes de recherche plus larges, au niveau Européen (FSO, SOLIBAM, DIVERSIFOOD, REVERSE), au niveau national (ProAbiodiv, COVALIENCE, MAPOD) ou alors au niveau local. Les préoccupations de certains producteurs du groupe de Dordogne font émerger de nouveaux axes de recherche, avec par exemple la recherche optimale de la densité de semis pour les variétés paysannes ou la question de l'efficacité de la sélection massale telle qu'appliquée jusqu'alors.

«C'est en 2010 qu'Armand a mis en place sa première parcelle d'expérimentation avec la variété Population Poromb. Cette variété lui a été confiée en fonction de ses types de sols et des indices de précocité qu'il avait l'habitude d'utiliser. Il a ressemé cette variété en 2011 à partir de ses propres semences et a entamé un protocole d'expérimentation sur l'impact de la sélection massale. [...] Il lui semble important de déterminer les impacts de la sélection sur les variétés, et ce en continuant les essais comme celui qu'il a déjà entamé chez lui. Les premières observations réalisées suscitent de nombreux questionnements chez Armand et de nouvelles pistes d'expérimentations sont à développer ». (Collectif 2015)

La comparaison d'une partie de la Population Poromb sélectionnée par Armand et une seconde partie non sélectionnée a été poussée pendant six ans. Les observations effectuées sur le terrain ne montrent pas de différences significatives (rendement et autres caractères phénotypiques) entre la souche sélectionnée et la souche non sélectionnée : ces résultats questionnèrent alors l'efficacité de la sélection telle qu'elle était conseillée par AgroBio Périgord et pratiquée par certains agriculteurs sélectionneurs du réseau.

Pour inciter la question de l'efficacité de la sélection, une expérimentation, appelée " essai version » a été mise en place en 2016 et 2017. Cette expérimentation visait à analyser le modèle de sélection d'un agriculteur en étudiant son discours, sa pratique et l'évolution phénotypique des variétés. Les résultats montrent un discours riche et complexe contenant de nombreuses règles de décisions mais avec peu d'objectifs de sélection formalisés. La mesure de la pratique de sélection consistait à étudier les différences entre une population et la portion de cette population sélectionnée par l'agriculteur : parmi 21 caractères phénotypiques étudiés, la sélection est légèrement différente de la population sur uniquement deux caractères : une de ces différences va à l'encontre d'un des objectifs de l'agriculteur. Enfin, l'étude de l'évolution des variétés a consisté à comparer, en station, une génération 2011 et une génération 2015 de 12 variétés Populations sélectionnées par l'agriculteur : cette comparaison montre, toutes 
variétés confondues, des générations 2015 plus grandes en gabarit de plante, plus tardives et avec légèrement moins de rendement: des résultats qui ne vont pas dans le sens des objectifs de l'agriculteur.

Pour donner suite à cette étude, les agriculteurs et les techniciens d'AgroBio Périgord décident de recadrer les objectifs de sélection avec plus de précision et de es décliner en un nombre restreint de critères de sélection. Ces critères de sélection doivent être facilement mesurables et parmi les plus héritables possible. La sélection sera focalisée sur ces caractères précis et sera aussi pratiquée par les agriculteurs à travers la sélection massale pour maximiser les différences entre la population visée.

Lors de cette étude, les bases de la génétique quantitative ont été mobilisées afin de formaliser l'étude de la sélection paysanne dans un cadre scientifique et technique robuste. La formalisation de la sélection paysanne dans ce cadre permet alors de développer des outils d'aide à la décision destinés aux agriculteurs sélectionneurs. L'animation de l'équipe biodiversité d'AgroBio Périgord porte notamment aujourd'hui vers l'accompagnement à la définition d'objectifs de sélection clairs et vers la proposition de méthodes de sélections personnalisées à chaque objectif. Dans le cadre du projet CASDAR COVALIENCE, un réseau d'expérimentations a été mis en place en 2018 sur 19 fermes afin d'éprouver l'efficacité de ces nouvelles méthodes de sélection.

\section{Conclusion}

36 Il est aujourd'hui difficile d'inventorier le nombre de fermes dans lesquelles du Maïs Population est cultivé, et de ce fait le nombre d'hectares cultivés au niveau national est inconnu.
«L'expérience menée sur la plate-forme par l'ensemble des agriculteurs investis dans le programme L'Aquitaine cultive la biodiversité est celle de pionniers. [...] La sélection à la ferme s'étend dans tout l'ouest à partir de l'expérience de "Bio d'Aquitaine ». [...] Le succès de la sélection de Populations à la ferme se mesure aujourd'hui par l'extension de l'expérience à tout l'ouest de la France, du Pays Basque à la Bretagne.[...] Le programme de "Bio d'Aquitaine » a contribué à développer le concept de sélection paysanne et en montrer l'efficacité» (Véronique Chable 2011) ${ }^{2}$.

$37 \mathrm{Au}$ démarrage, les agriculteurs contactaient AgroBio Périgord, qui était l'unique structure diffusant des variétés Population de maïs et un suivi était possible. Maintenant plus d'une quinzaine d'organisations relayent ce travail localement, animant des dynamiques collectives allant d'une dizaine jusqu'à parfois plus de 50 agriculteurs. De plus, même si elle est déconseillée, une diffusion hors collectif, de paysan à paysan, existe.

Cette perte de la comptabilisation exacte du nombre d'agriculteurs cultivant du Maïs Population en France n'empêche pas d'avoir un ordre d'idée en se basant sur les chiffres du passé (plus de 350 en 2012, plus de 700 en 2016...). Ceci laisse à penser que le millier de personnes qui ont un jour cultivé du maïs population a été largement atteint.

Sans savoir jusqu'où allait se développer cette aventure, en plus de 15 ans, en partant du bouche-à-oreille, cette initiative a eu un écho national et même une reconnaissance internationale. Elle a porté plus que les fruits attendus et continue à se développer et à vivre, sans forcément être en lien direct avec AgroBio Périgord. Les collectifs qui sont nés de cet essaimage sont tous connectés et un « Groupe National Maïs Population » a vu 
le jour en 2016. De plus, certains projets permettent de créer des espaces d'échanges «formels» entre collectifs (ProAbiodiv, Covalience, MIAM du maïs paysan dans mon assiette...) permettant à tous de s'inspirer des idées et fonctionnements de chacun (rédaction d'ouvrages, schématisation des fonctionnements...). Aujourd'hui, l'expérience de ces collectifs et les riches échanges entre tous apportent tout autant à AgroBio Périgord, qui découvre de nouveaux modes de fonctionnements autour du Maïs Population, apportant de l'eau à son moulin.

\section{BIBLIOGRAPHIE}

AgroBio Périgord 2003-2020 - L’Aquitaine cultive la Biodiversité. Programme régional d'expérimentation en population. Bilan, résultats, perspectives. Rapports annuels (Consultables en ligne : http://www.agrobioperigord.fr/).

Bio d'Aquitaine 2001 - Témoignage de Bertrand Lassaigne. In : L'Aquitaine cultive la Biodiversité, 2001-2011, 10 ans d'expériences et d'expérimentation sur les variétés paysannes de maïs et de tournesol. Autoédition, $127 \mathrm{p}$.

Collectif 2015 - Gérer collectivement la biodiversité cultivée. Etudes d'initiatives locales. Educagri éditions, $224 \mathrm{p}$.

FNAB, Bio d'Aquitaine \& CAB Pays de la Loire 2014 - En bio semons la diversité ! Recueil d'expériences du réseau FNAB sur les semences de population en grandes cultures. Autoédition, $51 \mathrm{p}$.

FRB 2011 - Gestion des ressources génétiques, Bertrand Lassaigne. Biodiversité, paroles d'acteurs :

Rencontres avec le Conseil d'Orientation Stratégique de la FRB. Autoédition, 383 p.

Réseau Semences Paysannes (RSP) 2014 - Les Maisons des Semences Paysannes. Regards sur la gestion collective de la biodiversité cultivée en France. Autoédition, 76 p.

Réseau Semences Paysannes (RSP), AgroBio Périgord \& Bio d'Aquitaine 2009 - Cahier technique : Variété paysannes de maïs et tournesol pour une agriculture écologique et économe. Autoédition, $120 \mathrm{p}$.

Site web : http://www.agrobioperigord.fr/produire-bio/biodiversite-cultivee

\section{NOTES}

1. D’après le "Réseau Semences Paysannes », les semences paysannes sont "des semences issues d'une population ou d'un ensemble de populations dynamiques reproductibles par le cultivateur, sélectionnées et multipliées avec des méthodes non transgressives de la cellule végétale et à la portée du cultivateur final, dans les champs, les jardins, les vergers conduits en agricultures paysanne, biologique ou biodynamique. Ces semences sont renouvelées par multiplications successives en pollinisation libre et/ou sélection massale, sans auto-fécondation forcée sur plusieurs générations. Elles sont librement échangeables dans le respect des droits d'usage définis par les collectifs qui les font vivre. » (Collectif 2015, voir chapitre 1.2 et Glossaire). 
2. Le CRB du Maïs de Montpellier à Mauguio est basé à L'UE Diascope de l'INRA. Il contient une des plus importantes collections nationales de Maïs Population. http://florilege.arcad-project.org/ $\mathrm{fr} / \mathrm{crb} / \mathrm{mais}$

3. RSP : Réseau Semences paysannes.

4. INRA : Institut Nationale de la Recherche Agronomique.

5. ITAB : Institut Technique de l'Agriculture Biologique.

6. https://eur-lex.europa.eu/legal-content/FR/TXT/PDF/?

uri=CONSIL:ST_8060_2018_REV_1\&qid=1525249294992\&from=FR

\section{RÉSUMÉS}

Depuis plus de 15 ans, un groupe d'agriculteurs de Dordogne se mobilise sur l'origine, la qualité et la diversité des variétés utilisées en agriculture biologique et durable. Cet article décrit la prise de conscience des agriculteurs d'Agrobio-Périgord et les actions mises en place qui permettent de se réapproprier les savoir-faire en sélection évolutive et en autoproduction de semences paysannes dans leurs fermes, notamment pour les populations de maïs. Ils répondent ainsi à leur souci de maintien de la biodiversité, d'autonomie, d'économie et d'adaptation à leurs modes de cultures. Agrobio-Périgord, au sein du programme «Cultivons la biodiversité en NouvelleAquitaine » œuvre à l'expérimentation, la sélection et la réappropriation des savoir-faire sur les variétés paysannes.

For more than 15 years, a group of farmers from Dordogne has been working on the origin, quality and diversity of crops used in organic and sustainable farming systems. This article describes the rising of awareness among Agrobio Périgord farmers and the actions implemented to recover traditional knowledge in evolutionary plant breeding and in self-production of seed saving and integrate them on their farms, in particular for maize. They thus tackle important questions about biodiversity conservation and farmers' autonomy, as well as savings and the adaptation of crops and practices to their farming methods. Within the "Cultivons la biodiversité en Nouvelle-Aquitaine" program, Agrobio Périgord works on experimentation, plant breeding and recovery of knowledge on peasant seeds.

Durante más de 15 años un grupo de agricultores de la Dordoña han venido trabajando sobre el origen, la calidad y la diversidad de las variedades utilizadas en los sistemas agrícolas de agricultura orgánica y sostenible. Este artículo describe la toma de conciencia de estos agricultores organizados en la asociación Agrobio Perigord, y las acciones que han implementado para recuperar los conocimientos sobre selección evolutiva y la autoproducción de semillas campesinas al interior de sus parcelas, especialmente para las poblaciones de maíz. Respondiendo con ello a su preocupación por mantener la biodiversidad, la autonomía, el ahorro y la adaptación de sus métodos de cultivo. Así, la asociación Agrobio Perigord trabaja, en el marco del programa "Cultivemos la biodiversidad en Nueva Aquitania », en la experimentación, selección y reapropiación de conocimientos sobre variedades campesinas. 
INDEX

Mots-clés : semences paysannes, AgroBio Périgord, sélection, agriculture biologique

Keywords : seed saving, AgroBio Périgord, plant breeding, organic agriculture

Palabras claves : semillas campesina, AgroBio Périgord, selección, agricultura biológica

\section{AUTEUR}

\section{AGROBIO PÉRIGORD}

Association de développement de l'agriculture biologique en Dordogne, 7 Impasse de la Truffe, 24430 Coursac, France 